\title{
Influence of surface contamination on the wettability of heat transfer surfaces
}

\author{
Eric Forrest ${ }^{a, *}$ \\ ecforre@sandia.gov \\ Roland Schulze \\ rkschulze@lanl.gov \\ Cheng Liu ${ }^{\mathrm{c}}$ \\ cliu@lanl.gov \\ David Dombrowski ${ }^{\text {b }}$ \\ ddombrow@lanl.gov \\ ${ }^{a}$ Primary Standards Laboratory, Sandia National Laboratories \\ P.O. Box 5800 \\ Albuquerque, NM 87185-0665, USA \\ ${ }^{\mathrm{b}}$ MST-6, Los Alamos National Laboratory \\ P.O. Box 1663 \\ Los Alamos, NM 87545, USA \\ ${ }^{\mathrm{c}}$ MST-8, Los Alamos National Laboratory \\ P.O. Box 1663 \\ Los Alamos, NM 87545, USA \\ *Corresponding author. Tel.: +1 505844 1377; fax: +1 5058444372 .
}

\begin{abstract}
The wettability of heat transfer surfaces plays an important role in liquid-vapor phase change phenomena, including boiling incipience, the critical heat flux, the Leidenfrost transition, and condensation. The influence of adsorbed surface contamination at the nanoscale, though seldom considered, can have a profound impact on wetting behavior. This study quantitatively investigates the impact of contaminant layer thickness on wettability. Various cleaning treatments are explored on zirconium and 6061 aluminum to determine the effect on contaminant and oxide layer thickness. Angle-resolved X-ray photoelectron spectroscopy can be used to measure the thickness of oxide and contaminant layers, which is then correlated to wettability by measuring the equilibrium contact angle. Results indicate that even after solvent cleaning, the contact angle of water on practical heat transfer surfaces is dominated by a hydrocarbon contaminant overlayer around five nanometers thick.
\end{abstract}




\title{
Influence of surface contamination on the wettability of heat transfer surfaces
}

\author{
Eric Forrest ${ }^{\mathrm{a}, *}$, Roland Schulze ${ }^{\mathrm{b}}$, Cheng Liu ${ }^{\mathrm{c}}$, David Dombrowski ${ }^{\mathrm{b}}$ \\ ${ }^{a}$ Primary Standards Laboratory, Sandia National Laboratories, Albuquerque, NM, USA \\ ${ }^{\mathrm{b}}$ MST-6, Los Alamos National Laboratory, Los Alamos, NM, USA \\ ${ }^{\mathrm{c}}$ MST-8, Los Alamos National Laboratory, Los Alamos, NM, USA \\ "Corresponding author. Tel.: +1 505844 1377; fax: +1 5058444372. \\ E-mail address: ecforre@sandia.gov (E. Forrest)
}

\begin{abstract}
The wettability of heat transfer surfaces plays an important role in liquid-vapor phase change phenomena, including boiling incipience, the critical heat flux, the Leidenfrost transition, and condensation. The influence of adsorbed surface contamination at the nanoscale, though seldom considered, can have a profound impact on wetting behavior. This study quantitatively investigates the impact of contaminant layer thickness on wettability. Various cleaning treatments are explored on zirconium and 6061 aluminum to determine the effect on contaminant and oxide layer thickness. Angle-resolved X-ray photoelectron spectroscopy can be used to measure the thickness of oxide and contaminant layers, which is then correlated to wettability by measuring the equilibrium contact angle. Results indicate that even after solvent cleaning, the contact angle of water on practical heat transfer surfaces is dominated by a hydrocarbon contaminant overlayer around five nanometers thick.
\end{abstract}

Keywords:

Wettability

Surface contamination

Boiling heat transfer

Critical heat flux

X-ray Photoelectron spectroscopy 


\section{Introduction}

Surface wettability influences nearly all aspects of liquid-vapor phase change at an interface, including nucleation, the critical heat flux (CHF), the Leidenfrost transition, and condensation. Early research qualitatively revealed that surface wetting could affect boiling phenomena. In his classic pool boiling study, Nukiyama observed that changes to the surface from electrolysis affected the boiling curves [1]. As early as 1964, Costello [2] and Gambill [3] noted the importance of capillary wicking, wettability, and deposit formation on burnout. Unfortunately, the resolution of surface measurement techniques was limited at the time, precluding the investigation of surface effects at sub-micron length scales.

The study of boiling and quenching in nanofluids $[4,5,6]$ and phase change on nanoengineered surfaces $[7,8]$ over the past decade has provided additional support that a strong link exists between wettability and two-phase phenomena. These and similar studies have also demonstrated that changes on nanometer-length scales are all that is required to alter wettability, and in turn, boiling and condensation behavior. The introduction of models incorporating contact angle provide more quantitative means to directly relate surface wettability to two-phase heat transfer, such as that proposed by Kandlikar for CHF [9]:

$$
\begin{gathered}
q_{C H F}^{\prime \prime}=h_{f g} \rho_{g}^{1 / 2}\left[\frac{1+\cos \theta_{r}}{16}\right]\left[2 / \pi+\frac{\pi}{4}\left(1+\cos \theta_{r}\right) \cos \phi\right]^{1 / 2} \\
\times\left[\sigma g\left(\rho_{f}-\rho_{g}\right)\right]^{1 / 4}
\end{gathered}
$$

However, the impact of surface cleanliness on boiling and condensation behavior still needs to be explored.

Surface contamination is unavoidable on a practical heat transfer surface and will influence wettability. Mantel showed a strong dependence of surface energy (and therefore wettability) on organic contaminant thickness for stainless steels [10]. Even deionized water will contain some contaminants, which can also deposit on heat transfer surfaces. Thus, attempting to achieve a clean room-type setting in a heat transfer laboratory would prove quite difficult. With increasing focus on wettability effects in two-phase heat transfer, it is important to understand the nature of surface layers on typical heat transfer surfaces, and how these layers affect wetting behavior. Modern surface science techniques, such as angle-resolved X-ray 
photoelectron spectroscopy (AR-XPS), can be utilized to identify and measure surface layers on heat transfer surfaces.

This study quantitatively relates wettability to the thickness of surface layers on zirconium and 6061 aluminum. These materials are encountered as heat transfer surfaces in a variety of systems, including heat exchangers and as nuclear fuel cladding. The study also demonstrates the effectiveness of different cleaning treatments on removing these contaminant layers, and the corresponding effect on water droplet contact angle.

\section{Experimental}

Several surface conditions, with varying degrees of contamination, were investigated on zirconium and 6061 aluminum foils. The levels of cleanliness and associated cleaning treatment are summarized in Table 1, with two nominally identical coupons being prepared for each case. In heat transfer studies, surfaces are typically prepared by cleaning with solvents. The solvent cleaning process employed here consisted of immersion in an ultrasonic bath of ACS Reagent grade acetone for 10 minutes, followed by rinses with ultrapure ethanol and deionized water, and final drying with UHP compressed nitrogen. The chemical etching cleaning treatments are specific to each metal, and involved ultrasonic cleaning in a detergent solution followed by multi-step etching in nitric acid/ammonium bifluoride and/or sodium hydroxide. The different cleaning processes for 6061 aluminum and zirconium are based on established techniques to achieve uniform etches and repeatable surface conditions, and are described in further detail in reference [11]. For surfaces which were intentionally contaminated, a highly refined mineral oil was applied.

Composition and thickness of contaminant and oxide layers were determined quantitatively using AR-XPS. XPS relies on the photoelectric effect, in which an incident photon results in ejection of an electron from its orbital. Samples are irradiated with soft monoenergetic X-rays, with $\mathrm{Mg} \mathrm{K \alpha}$ or $\mathrm{Al} \mathrm{K} \alpha$ typically being used [12]. The photoelectron kinetic energy is measured, from which the binding energy is calculated. XPS can therefore determine elemental composition and concentration at the surface, along with the chemical state, from slight shifts in the binding energy. Angle-resolved XPS allows for measurement of surface layer thicknesses, typically on the order of a few hundred $\AA$ or less. By varying the sample angle $\left(90^{\circ}, 45^{\circ}\right.$, and $\left.15^{\circ}\right)$, depth profiling was performed. Details of the technique are provided in reference [11]. 
Table 1: Summary of Surface Conditions Investigated.

\begin{tabular}{|c|c|}
\hline SURFACE CONDITION & DESCRIPTION \\
\hline As-received & $\begin{array}{l}\text { Removal of any visible dust with DI water and a task } \\
\text { wipe, and dried with clean, compressed nitrogen. }\end{array}$ \\
\hline As-received with solvent clean & $\begin{array}{l}\text { Immersion for } 10 \text { minutes in ultrasonic bath of } \\
\text { acetone, followed by rinse with ethanol, rinse with DI } \\
\text { water, and blown dry with clean compressed } \\
\text { nitrogen. }\end{array}$ \\
\hline Chemically etched & $\begin{array}{l}\text { Ultrasonic cleaning with detergent followed by multi- } \\
\text { step etching in nitric acid/ammonium bifluoride } \\
\text { and/or sodium hydroxide. Specific to each metal. }\end{array}$ \\
\hline Mild contamination & $\begin{array}{l}\text { Following chemical etching treatment, surfaces were } \\
\text { coated with light hydrocarbon-based oil that was } \\
\text { wiped clean with a dry task wipe. }\end{array}$ \\
\hline $\begin{array}{l}\text { Mild contamination } \\
\text { followed by solvent clean }\end{array}$ & $\begin{array}{l}\text { Following chemical etching treatment and subsequent } \\
\text { intentional oil contamination, surfaces were cleaned } \\
\text { using the standard solvent cleaning process. }\end{array}$ \\
\hline
\end{tabular}

In this study, high resolution spectra were collected for each chemical species at every take-off angle (TOA) for a minimum of 60 minutes. Peak analysis was performed using Physical Electronics MultiPak software. Surface layer thicknesses were calculated using the software package QUASES-ARXPS. Figure 1 illustrates the three-layer model used in QUASES-ARXPS for computing thicknesses based on the measured AR-XPS intensity data. In reality, surface layers on metals exposed to atmosphere are somewhat more complex, as described by Castle [13], but the three-layer model is a reasonable approximation for metals that form thin, protective native oxides. The electron mean free paths through the materials of interest were obtained from the NIST Standard Reference Database 71 [14]. Varying the take-off angle yielded different path lengths, allowing for calculation of layer thickness from relative peak intensities due to attenuation. Thicknesses were calculated in the software by minimizing the weighted root mean square (RMS) error between the model and measured data. Wettability was characterized on the 6061 aluminum and zirconium surfaces using contact angle measurements. Sessile drop measurements were performed at a minimum of three locations on each surface to determine the equilibrium contact angle.

Both XPS and contact angle measurements were performed one week after the cleaning treatments, to allow native surface oxide and contaminant layers to reach an equilibrium thickness. The one week aging period was guided by prior studies investigating the kinetics of re-contamination on metal and oxide surfaces $[15,16]$, suggesting a period of hours to several 
days to achieve steady-state contact angles, depending on the substrate material. Between cleaning and measurements, surfaces were stored in ultra-high vacuum (UHV) foil in a desiccator box exposed to laboratory air, ensuring removal of any moisture which could affect both XPS and contact angle measurements. Storage in UHV foil in air has been shown to prevent gross contamination (dust, particulates, etc.) of recently cleaned surfaces [17].

Uncertainties for measured and calculated quantities were determined using methods recommended by the ASME and ISO [18, 19]. Uncertainties are reported at the $95 \%$ confidence level. For a discussion of typical sources of uncertainty in surface science techniques such as XPS, refer to [20] and [21]. The accuracy of the QUASES-ARXPS approach to calculating layer thicknesses has been validated in prior studies [22, 23]. In this study, relative normalization was used in QUASES-ARXPS, with peak intensity values being weighted by their respective uncertainties.

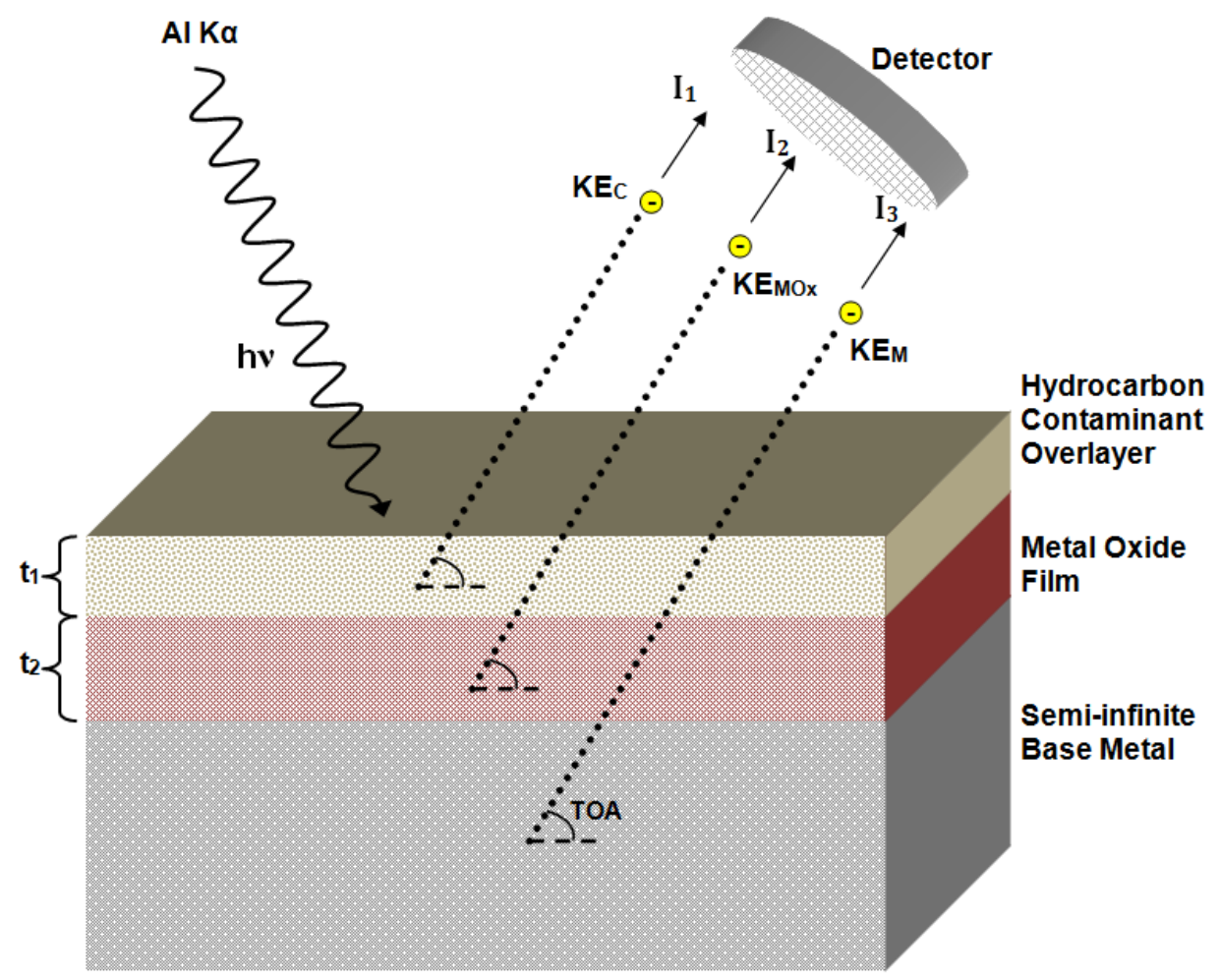

Figure 1: Three-Layer Model Used to Compute Thickness of Oxide and Contaminant Overlayers.

\section{Results and Discussion}

Figure 2 shows typical XPS survey spectra for 6061 aluminum following various cleaning treatments. Note that the intensity (area under peak relative to background) should be 
used as a basis for comparison between measurements. In Figure 2, note the distinct difference in surface composition achieved with various cleaning treatments on aluminum 6061. While 6061 is alloyed with magnesium and silicon, the large presence of these elements at the surface, giving a higher signal than aluminum itself, seems to indicate the presence of a protectant or contaminant from the production process, which solvent cleaning does not remove. Although not shown, it was also discovered during this study that as-received zirconium foil possessed sub-stoichiometric surface oxides, along with the possible presence of zirconium carbide on the surface. Only the chemical etching process removed these layers and fostered the formation of a stoichiometric, passive oxide layer on the zirconium surface.

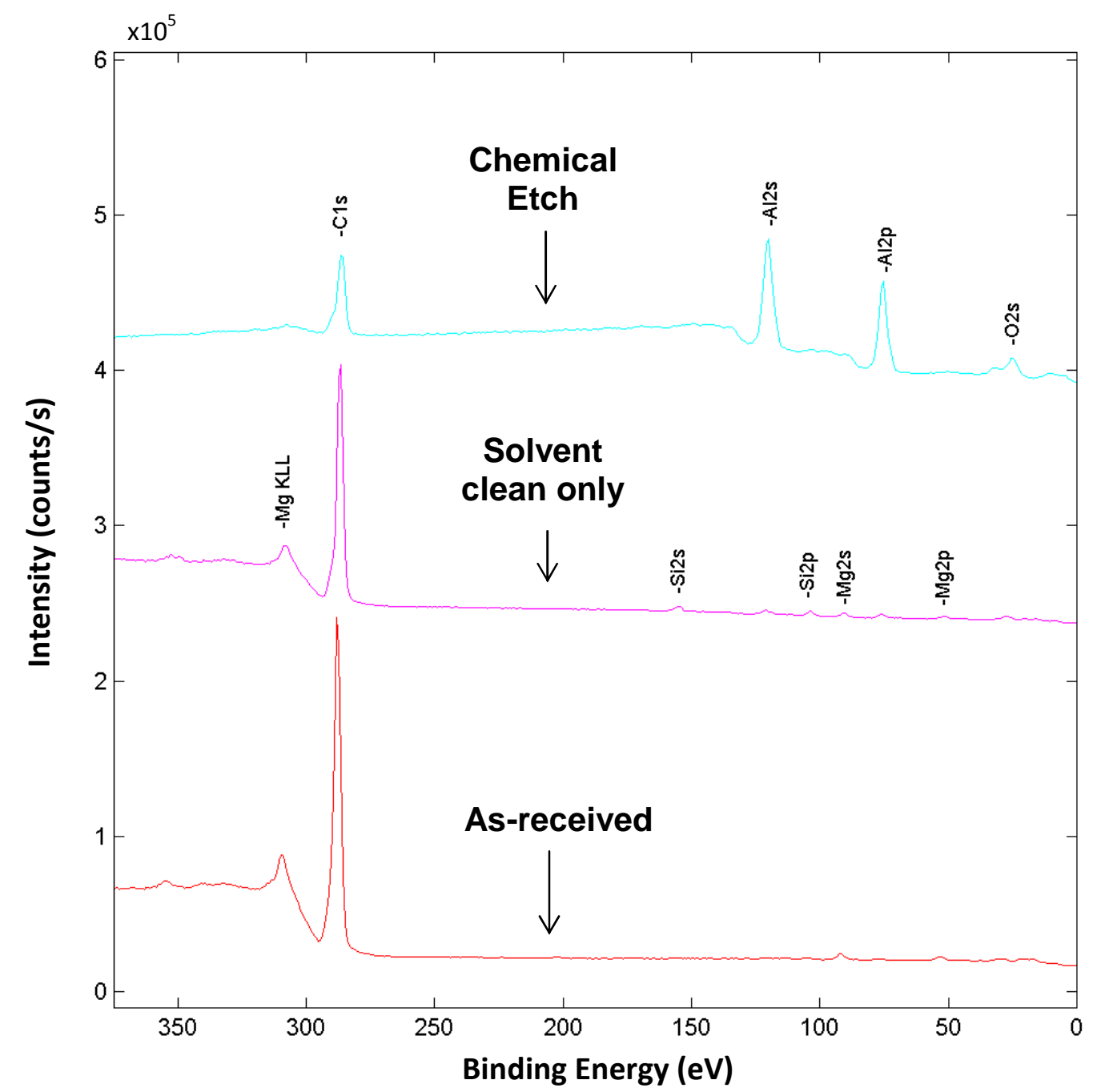

Figure 2: XPS Survey Spectra of 6061 Foils Using Al Monochromatic Source at TOA=45․ Bottom: As-received; Middle: As-received with solvent clean only; Top: Cleaned with chemical etch. Note that the solvent cleaning still leaves a significant amount of hydrocarbon on the surface. 
High resolution XPS spectra were collected for each surface at the relevant carbon, metal, and metal oxide energies for three TOA's. Figure 3 shows one such spectrum, demonstrating the observable shift in binding energy from the metal to the metal oxide signal at different TOA's. These results were used to compute the oxide and hydrocarbon contaminant overlayer thickness. For the as-received surfaces, the hydrocarbon contaminant overlayer was so thick that signal could not be obtained from the underlying metal nor the metal oxide. Results are summarized in Table 2 for cases where sufficient signal was obtained from the underlying metal and metal oxide such that the three layer calculation could be applied. Uncertainty in the calculated thickness is at the $95 \%$ confidence level and captures the random uncertainty determined from the counting process. Thicker overlayers typically led to larger uncertainties in the calculated thickness due to a reduction in counts from each layer.

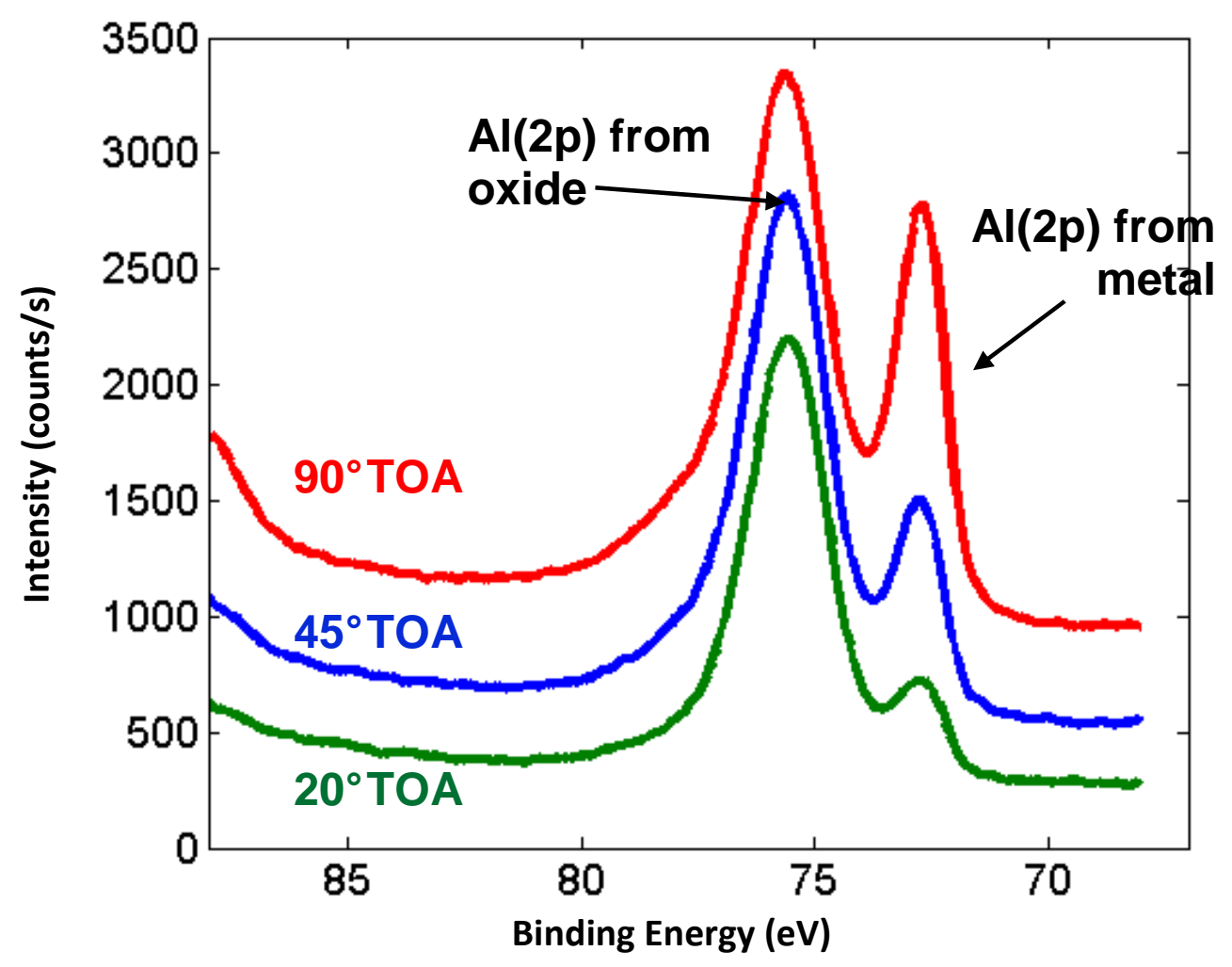

Figure 3: High Resolution XPS Spectra for 6061 Aluminum at Different TOA's. The binding energy shift between the metal and metal oxide is measurable, and the relative intensity change at different TOA's is the principle enabling calculation of layer thickness. 
Table 2: Summary of Oxide Layer and Hydrocarbon Layer Thicknesses Measured on Zirconium and 6061 Aluminum Foils of Varying Degrees of Cleanliness.

\begin{tabular}{l|cc}
\hline \multicolumn{1}{c|}{ SPECIMEN } & $\begin{array}{c}\text { OXIDE } \\
\text { THICKNESS } \\
\left(\mathrm{Al}_{2} \mathrm{O}_{3} \text { or ZrO }\right)\end{array}$ & $\begin{array}{c}\text { HYDROCARBON } \\
\text { THICKNESS }\end{array}$ \\
\hline $\begin{array}{l}\text { Al 6061, As-received with } \\
\text { Solvent Clean Only }\end{array}$ & $51 \pm 4 \AA$ & $54 \pm 4 \AA$ \\
Al 6061, Chemical Etch & $45 \pm 3 \AA$ & $4.8 \pm 0.6 \AA$ \\
Al 6061, Mild Contamination & $36 \pm 3 \AA$ & $94 \pm 4 \AA$ \\
$\begin{array}{l}\text { Al 6061, Mild Contamination } \\
\text { followed by solvent clean }\end{array}$ & $37 \pm 1 \AA$ & $13 \pm 0.8 \AA$ \\
& & \\
Zr, Chemical Etch & $57 \pm 1 \AA$ & $8.5 \pm 1 \AA$ \\
Zr, Mild Contamination & $77 \pm 3 \AA$ & $170 \pm 19 \AA$ \\
\hline
\end{tabular}

Equilibrium contact angles for deionized water on the zirconium and 6061 aluminum surfaces are plotted against hydrocarbon contaminant layer thickness in Figure 4. Note that contact angle increases (wettability decreases) for increasing contaminant layer thickness. This relates to a decrease in the surface energy of the solid with increasing hydrocarbon contaminant layer thickness. High surface energies are typically associated with clean metals, inorganic compounds such as metal oxides, intermetallic oxides, diamond, etc. Low surface energies are usually associated with polymers and hydrocarbons. By virtue of their high surface energy, clean metals will not remain clean when exposed to the environment due to the spontaneous adsorption of organic contaminants of low surface energy onto the high surface energy substrate to minimize the surface free energy of the system [24]. Studies indicate an adsorption phenomenon exists where specific metal and metal oxide surfaces have their own characteristic rate and amount of adsorption [10]. Typical surface free energies corresponding to surface layers on metal exposed to atmosphere are provided in Table 3. Note that experimentally reported surface energies for pure aluminum and pure zirconium metals are $1160 \mathrm{~mJ} / \mathrm{m}^{2}$ and $2000 \mathrm{~mJ} / \mathrm{m}^{2}$, respectively [25]. 
Table 3: Typical Surface Free Energies Corresponding to Contaminant Layers on a Metal Surface. Adapted from Ref. [13].

\begin{tabular}{l|c}
\hline \multicolumn{1}{c|}{ SURFACE } & $\begin{array}{c}\text { SURFACE FREE ENERGY } \\
\left(\mathbf{m J} / \mathbf{m}^{\mathbf{2}}\right)\end{array}$ \\
\hline Organic Hydrocarbons & $\sim 20$ \\
Organic Polymers & $\sim 20-30$ \\
Metal Oxides & $200-300$ \\
Metals & $1000-5000$ \\
\hline
\end{tabular}

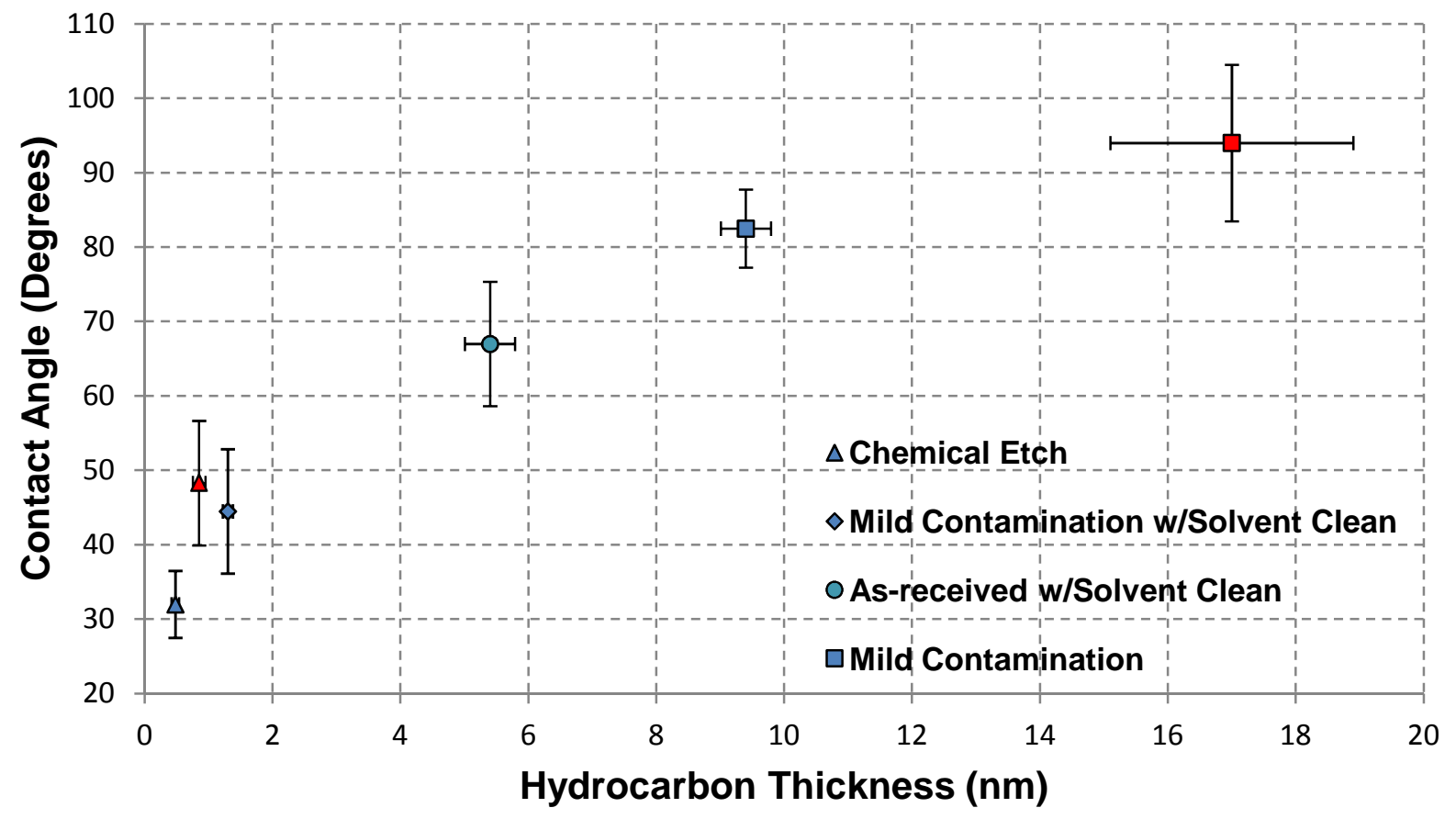

Figure 4: Sessile Drop Contact Angle of Water on 6061 Aluminum and Zirconium Surfaces as a Function of Adsorbed Hydrocarbon Contaminant Overlayer Thickness. Blue and red markers represent $6061 \mathrm{Al}$ and $\mathrm{Zr}$ substrates, respectively.

The anticipated influence of each surface condition and cleaning treatment on the critical heat flux can be predicted using equation (1). While the force balance in Kandlikar's equation relies on the receding contact angle, the goniometer setup employed could only perform equilibrium contact angle measurements. However, the foils studied were very smooth 
$\left(R_{a}<0.1 \mu \mathrm{m}\right)$, and Neumann [26] has shown that at this roughness scale, roughness has no effect and the contact angle hysteresis is negligible (i.e., the difference between advancing, equilibrium, and receding contact angles is small). Figure 5 plots the predicted value of the critical heat flux for pool boiling of saturated water at atmospheric pressure. As seen in the figure, the level of surface cleanliness is expected to have a substantial effect on CHF due to the change in contact angle.

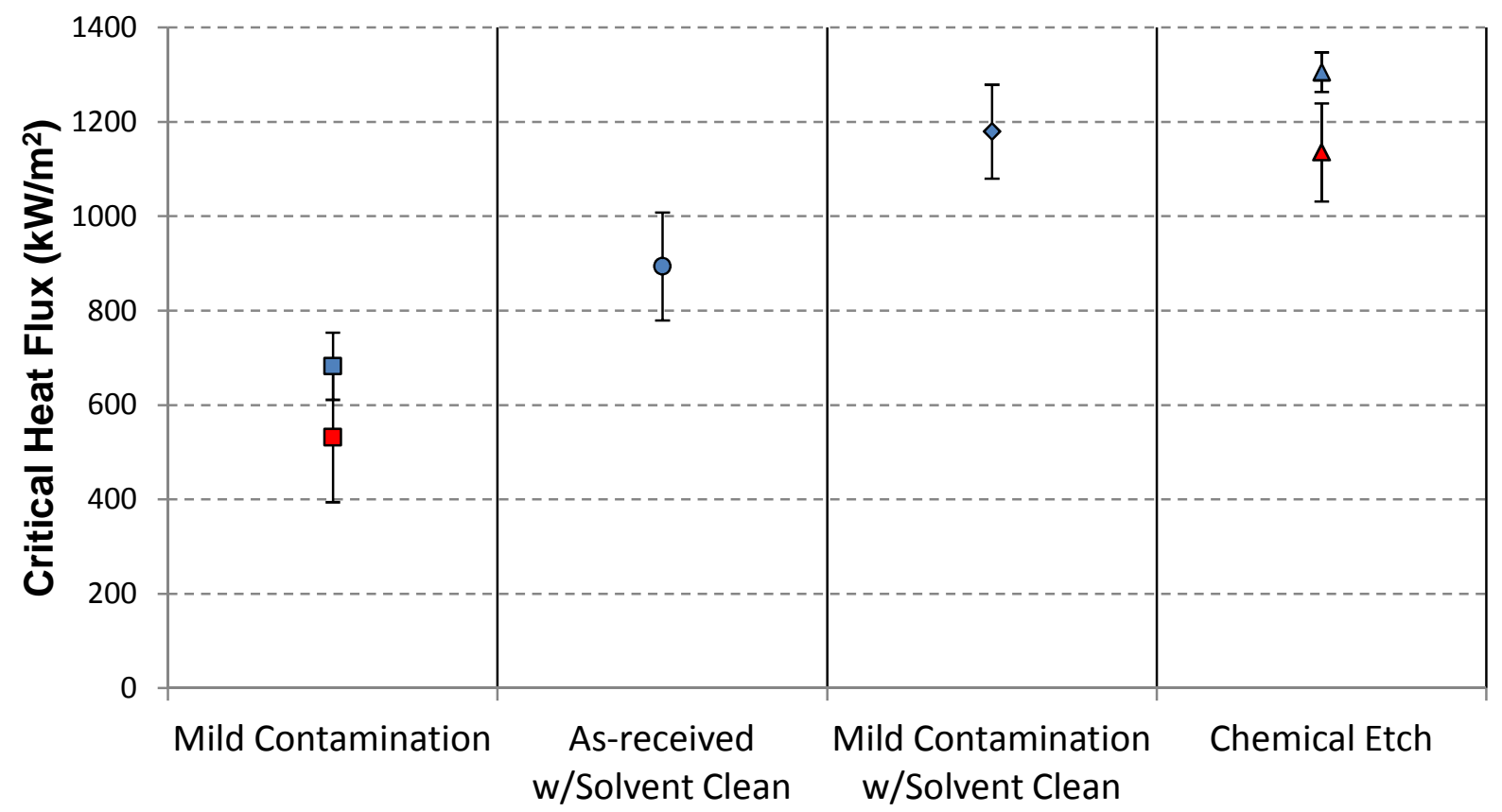

Figure 5: Predicted Effect of Surface Contamination on the Pool Boiling Critical Heat Flux of Saturated Water at Atmospheric Pressure. Blue and red markers represent $6061 \mathrm{Al}$ and $\mathrm{Zr}$ substrates, respectively.

The work by Athanase Dupré on adhesion between two immiscible liquids [27] resulted in the following relation:

$$
W_{A B}=\gamma_{A}+\gamma_{B}-\gamma_{A B}
$$

where the subscripts $A$ and $B$ denote each liquid. Dupré's result may be combined with Young's equation [28] to obtain what is commonly referred to as the Young-Dupré equation:

$$
W_{s l}=\sigma[1+\cos (\theta)]
$$


where $W_{s l}$ is the work of adhesion per unit surface area, and the spreading pressure, $\pi_{e}=\gamma_{s^{-}} \gamma_{s v}$ has been neglected, i.e. $\gamma_{s}=\gamma_{s v}$ and $\gamma_{l}=\gamma_{l v}=\sigma$. Note that the units of surface energy $\left(\mathrm{mJ} / \mathrm{m}^{2}\right)$ are equivalent to that of surface tension $(\mathrm{mN} / \mathrm{m})$. The work of adhesion for water at room temperature and atmospheric pressure on the surfaces studied here is plotted in Figure 6.

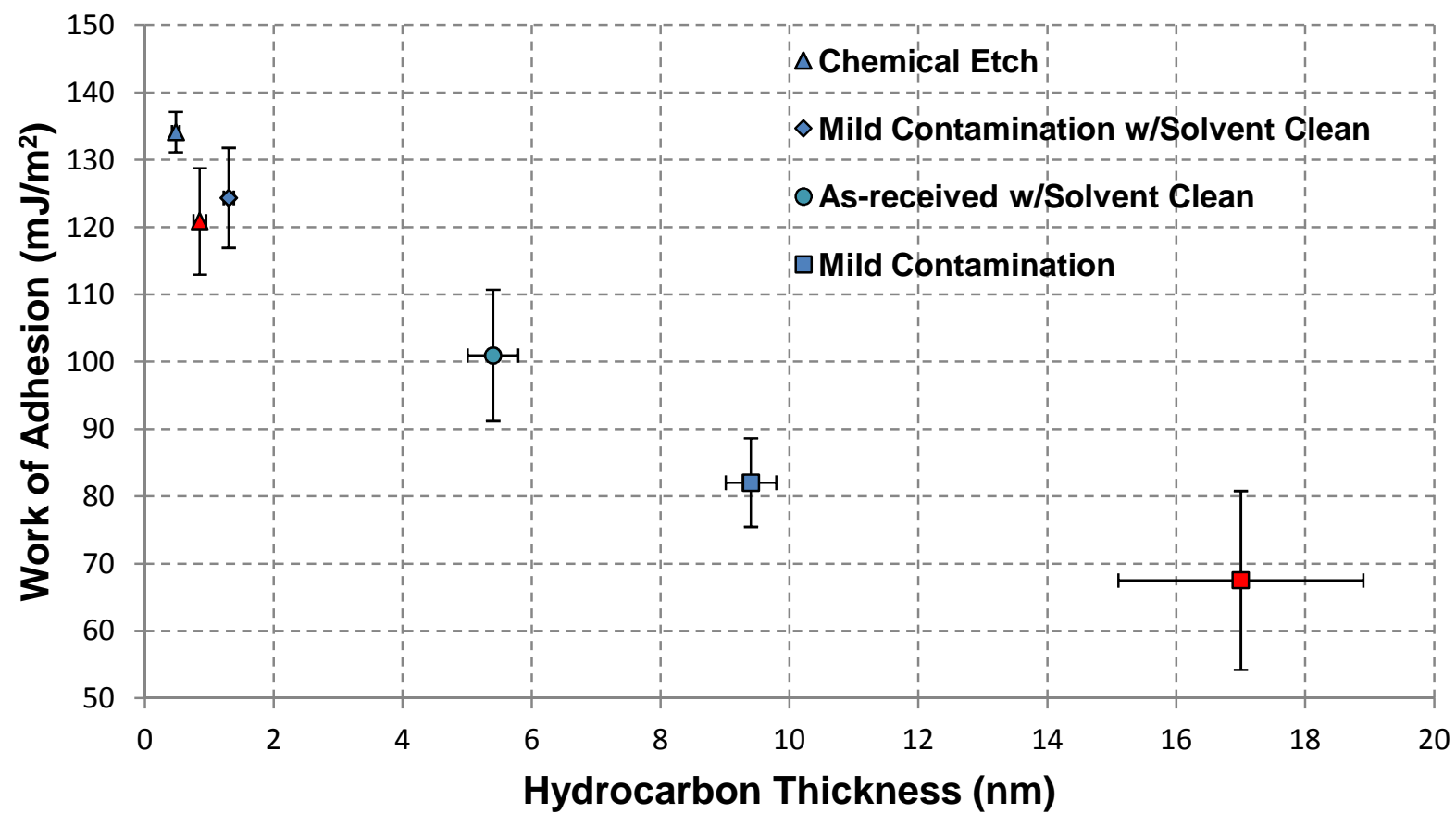

Figure 6: Work of Adhesion for Deionized Water versus Hydrocarbon Contaminant Thickness for Al 6061 and Zr Surfaces. Blue and red markers represent $6061 \mathrm{Al}$ and $\mathrm{Zr}$ substrates, respectively.

The surface tensions, or surface energies, may be broken into two components: a nonpolar dispersive element representing the effect of Lifshitz-van der Waals forces, which include Debye, Keesom, and London dispersion interactions, and a polar element representing hydrogen bonding-type interactions [29]:

$$
\gamma=\gamma^{D}+\gamma^{P}
$$

The polar component of the surface energy can be treated as Lewis acid-base interactions, whose constituents are not additive. However, when the spreading pressure is negligible, it has been shown experimentally that for certain situations the work of adhesion can be reasonably related to the surface energy by [10]: 


$$
\frac{W_{s l}}{2 \sqrt{\gamma_{l}^{D}}}=\left(\frac{\gamma_{l}^{P}}{\gamma_{l}^{D}}\right)^{1 / 2} \sqrt{\gamma_{s}^{P}}+\sqrt{\gamma_{s}^{D}}
$$

where for water at room temperature and atmospheric pressure, $\gamma_{l}=72.6 \mathrm{~mJ} / \mathrm{m}^{2}, \gamma_{l}^{D}=21.6 \mathrm{~mJ} / \mathrm{m}^{2}$, and $\gamma_{l}^{P}=51.0 \mathrm{~mJ} / \mathrm{m}^{2}$. It is important to stress that equation (5) is formulated assuming that the polar interaction constituents may be combined by a geometric mean, which has no theoretical basis and for this reason has been criticized [30]. In a more rigorous and theoretically correct approach, the polar and nonpolar solid surface tension components would be determined by solving a system of three equations by measuring contact angles for three different liquids, two of which must be polar and hydrogen-bonding [29].

The separation distance between the liquid water and the metal oxide is effectively the thickness of the hydrocarbon overlayer, which is assumed apolar. If the water were in direct contact with either the metal oxide or metal surface, perfect wetting would be expected [31]. For decades there was significant debate over whether clean, oxygen-free metal surfaces were perfectly wetting [32] or inherently non-wetting [33]. This study lends support to the former hypothesis, indicating that measured contact angle values in the range of $50^{\circ}$ to $85^{\circ}$ on "clean" metal surfaces [33] are actually a result of residual adsorbed contaminants, which are difficult to remove.

The energy associated with hydrogen bonding decays rapidly with distance. The work of adhesion due to the polar component decays as:

$$
W_{s l}^{P} \sim e^{\left(\delta_{0}-\delta\right) / \lambda}
$$

where $\delta_{0}=1.58 \AA$, and for water $\lambda \approx 2 \AA$ [34]. While the van der Waals potential between neighboring molecules has a $1 / d^{6}$ dependence, and therefore also falls off rapidly with increasing separation, the van der Waals potential between macroscopic bodies in the condensed state decays much more slowly with distance and is geometry dependent, as postulated by Hamaker [35] and further demonstrated by Lifshitz [36]. The Lifshitz-van der Waals forces exhibit longer range behavior, with the decay between two semi-infinite slabs being:

$$
W_{s l}^{D} \sim \frac{1}{\delta^{2}}
$$


for separations less than $100 \AA$, whereas at greater separations, retardation results in a decrease inversely proportional to $\delta^{3}$ [34]. Therefore, the Lifshitz-van der Waal interaction dominates with increasing hydrocarbon contaminant layer.

\section{Conclusions}

AR-XPS measurements have demonstrated the presence of hydrocarbon contaminant overlayers on practical surfaces of zirconium and 6061 aluminum. The equilibrium contaminant layer thickness can be reduced with various cleaning methods, but even the best solvent cleaning will leave behind over $5 \mathrm{~nm}$ of adsorbed hydrocarbon. Chemical etching processes were developed for zirconium and 6061 aluminum to yield a stable reduction in the hydrocarbon contaminant overlayer. The contact angle of deionized water was measured on the surfaces following various cleaning treatments, and correlated to hydrocarbon layer thickness. Results indicate that for practical heat transfer surfaces, the wetting process is dominated by an adsorbed hydrocarbon contaminant overlayer. However, wettability is not the sole surface parameter influencing liquid-vapor phase change, and temperature effects on contaminant layers require further investigation. Nonetheless, intensifying interest in the link between surface effects and liquid-vapor phase change phenomena necessitates increased consideration of cleaning treatments and the associated effects of adsorbed contaminant layers on heat transfer surfaces.

\section{Acknowledgements}

Randy Edwards of MST-6 is gratefully acknowledged for his assistance with the surface cleaning procedure. Jacopo Buongionro, Lin-Wen Hu, Thomas McKrell, Nishant Patel, Laura Elterman, Roger Burton, and Eric Detlefs are kindly acknowledged for reviewing the manuscript. This research was performed under appointment to the U.S. Department of Energy Nuclear Nonproliferation Safeguards Graduate Fellowship Program sponsored by the National Nuclear Security Administration's Office of Nonproliferation and International Security. Sandia National Laboratories is a multi-program laboratory managed and operated by Sandia Corporation, a wholly owned subsidiary of Lockheed Martin Corporation, for the U.S. Department of Energy's National Nuclear Security Administration under contract DE-AC04- 
94AL85000. This publication has been approved for unlimited public release, SAND2014$20008 \mathrm{~J}$.

\section{Nomenclature}

$\begin{array}{ll}C_{s, f} & \text { surface-fluid interaction constant } \\ g & \text { acceleration due to gravity }\left(\mathrm{m} / \mathrm{s}^{2}\right) \\ h_{f g} & \text { latent heat of vaporization }(\mathrm{J} / \mathrm{kg}-\mathrm{K}) \\ I & \text { intensity (counts/s) } \\ R_{a} & \text { arithmetic roughness }(\mu \mathrm{m}) \\ t & \text { thickness }(\AA \text { or } \mathrm{nm}) \\ q^{\prime \prime} & \left.\text { heat flux (W/m } \mathrm{m}^{2}\right) \\ W & \text { work of adhesion }\left(\mathrm{mJ} / \mathrm{m}^{2}\right)\end{array}$

Greek Symbols

$\gamma$

Surface energy $\left(\mathrm{mJ} / \mathrm{m}^{2}\right)$

$\delta$

separation or thickness of separating layer ( $\AA$ or nm)

$\theta$

contact angle (degrees)

$v$

frequency $(1 / \mathrm{s})$

$\pi_{e}$

spreading pressure $(\mathrm{mN} / \mathrm{m})$

$\rho$

density $\left(\mathrm{kg} / \mathrm{m}^{3}\right)$

$\sigma$

surface tension $(\mathrm{mN} / \mathrm{m})$ 
$\phi \quad$ heater orientation angle

\section{Subscripts/Superscripts}

$0 \quad$ in van der Waals contact

$1 \quad$ layer 1

2

layer 2

A

liquid A

$B$

liquid B

c

carbon

CHF

critical heat flux

$D$

dispersive, or Lifshitz-van der Waals

$f$

saturated liquid

$g$

saturated vapor

$H C$

hydrocarbon

$l$

liquid

M

metal

$M O_{x}$

metal oxide

$P$

polar, or hydrogen bonding

$r$

receding

$S$

solid 


\section{References}

[1] S. Nukiyama, The maximum and minimum values of the heat Q transmitted from metal to boiling water under atmospheric pressure, International Journal of Heat and Mass Transfer 9 (1966) 1419-1433. (Reprint from Journal of Japanese Society of Mechanical Engineers 37 (1934) 367-374)

[2] C.P Costello, W.J. Frea, The roles of capillary wicking and surface deposits in the attainment of high pool boiling burnout heat fluxes, A.I.Ch.E. Journal 10 (3) (1964) 393398.

[3] W. Gambill, An experimental investigation of the inherent uncertainty in pool boiling critical heat fluxes to saturated water, A.I.Ch.E. Journal 10 (4) (1964) 502-508.

[4] S.J. Kim, I.C. Bang, J. Buongiorno, L.W. Hu, Surface wettability change during pool boiling of nanofluids and its effect on critical heat flux, International Journal of Heat and Mass Transfer 50 (19-20) (2007) 4105-4116.

[5] J.S. Coursey, J. Kim, Nanofluid boiling: the effect of surface wettability, International Journal of Heat and Fluid Flow 29 (6) (2008) 1577-1585.

[6] H. Kim, G. DeWitt, T. McKrell, J. Buongiorno, L.W. Hu, On the quenching of steel and zircaloy spheres in water-based nanofluids with alumina, silica, and diamond nanoparticles, International Journal of Multiphase Flow 35 (5) (2009) 427-438.

[7] E. Forrest, E. Williamson, J. Buongiorno, L.W. Hu, M. Rubner, R. Cohen, Augmentation of nucleate boiling heat transfer and critical heat flux using nanoparticle thin-film coatings, International Journal of Heat and Mass Transfer 53 (1-3) (2010) 58-67.

[8] N. Miljkovic, R. Enright, E.N. Wang, Effect of droplet morphology on growth dynamics and heat transfer during condensation on superhydrophobic nanostructured surfaces, ACS Nano 6 (2) (2012) 1776-1785.

[9] S.G. Kandlikar, A theoretical model to predict pool boiling CHF incorporating effects of contact angle and orientation, Journal of Heat Transfer 123 (2001) 1071-1079.

[10] M. Mantel, J.P. Wightman, Influence of the surface chemistry on the wettability of stainless steel, Surface and Interface Analysis 21 (1994) 595-605.

[11] R. Edwards, M.A. Hill, R.K. Schulze, Mid-year report FY2011 Convert Program LEU fabrication process cleaning study process certification for material surface cleaning for bonded LEU fuel composite including AFIP-7 LEU coupons, LA-UR-11-02854, May 2011.

[12] J.F. Moulder, W.F. Stickle, P.E. Sobol, K. Bomben, Handbook of X-ray Photoelectron Spectroscopy, $2^{\text {nd }}$ Edition, Perkin-Elmer Corporation, Eden Prairie, MN, 1992. 
[13] J.E. Castle, The composition of metal surfaces after atmospheric exposure: an historical perspective, The Journal of Adhesion 84 (2008) 368-388.

[14] National Institute of Standards and Technology, NIST Electron Inelastic-Mean-Free-Path Database: Version 1.2, http://www.nist.gov/srd/nist71.cfm.

[15] D.F. O'Kane, K.L. Mittal, Plasma cleaning of metal surfaces, Journal of Vacuum Science and Technology 11 (3) (1974) 567-569.

[16] D.J. Preston, N. Miljkovic, J. Sack, R. Enright, J. Queeney, E.N. Wang, Effect of hydrocarbon adsorption on the wettability of rare earth oxide ceramics, Applied Physics Letters 105 (011601) (2014) 1-5.

[17] C. Scheuerlein, M. Taborelli, The assessment of metal surface cleanliness by XPS, Applied Surface Science 252 (2006) 4279-4288.

[18] Measurement uncertainty, ANSI/ASME PTC 19.1- 1985 Part I, 1986.

[19] Working Group 1 of the Joint Committee for Guides in Metrology, Evaluation of measurement data- guide to the expression of uncertainty in measurement, JCGM 100:2008- GUM 1995 with minor corrections, ISO, BIPM, IEC, IFCC, ILAC, IUPAC, and OIML, September 2008.

[20] P.J Cumpson, M.P. Seah, Random uncertainties in AES and XPS: I: uncertainties in peak energies, intensities and areas derived from peak synthesis, Surface and Interface Analysis 18 (1992) 345-360.

[21] J.E. Fulghum, Determination of overlayer thickness by angle-resolved XPS: a comparison of algorithms, Surface and Interface Analysis 20 (1993) 161-173.

[22] T.S. Lassen, S. Tougaard, A. Jablonski, Practical correction procedures for elastic scattering effects in ARXPS, Surface Science 481 (2001) 150-162.

[23] B.S. Semak, C. van der Marel, S. Tougaard, Comparison of the Tougaard, ARXPS, RBS and ellipsometry methods to determine the thickness of thin $\mathrm{SiO}_{2}$ layers, Surface and Interface Analysis 33 (2002) 238-244.

[24] E. Ebnesajjad, Handbook of Adhesives and Surface Preparation: Technology, Applications and Manufacturing, Elsevier, Burlington, MA, 2011, p. 23.

[25] L. Vitos, A.V. Ruban, H.L. Skriver, J. Kollár, The surface energy of metals, Surface Science 411 (1998) 186-202.

[26] A.W. Neumann, R.J. Good, Thermodynamics of contact angles I. Heterogeneous solid surfaces, Journal of Colloid and Interface Science 38 (2) (1972) 341-358. 
[27] M.A. Dupré, Théorie Mécanique de La Chaleur, Gauthier-Villars, Imprimeur Libraire de L'École Polytechnique, Paris, France, 1869.

[28] T. Young, An essay on the cohesion of fluids, Philosophical Transactions of the Royal Society of London 95 (1805) 65-87.

[29] C.J. van Oss, R.J. Good, M.K. Chaudhury, Additive and nonadditive surface tension components and the interpretation of contact angles, Langmuir 4 (1988) 884-891.

[30] R.J. Good, Contact angle, wetting, and adhesion: a critical review, Journal of Adhesion Science and Technology 6 (12) (1992) 1269-1302.

[31] M.E. Schrader, Wettability of clean metals, Journal of Colloid and Interface Science 100 (2) (1984) 372-380.

[32] K.W. Bewig, W.A. Zisman, The wetting of gold and platinum by water, The Journal of Physical Chemistry 69 (12) (1965) 4238-4242.

[33] F.M. Fowkes, Attractive forces at interfaces, Industrial and Engineering Chemistry 56 (12) (1964) 40-52.

[34] C.J. van Oss, M.K. Chaudhury, R.J. Good, Interfacial Lifshitz-van der Waals and polar interactions in macroscopic systems, Chemical Reviews 88 (1988) 927-941.

[35] H.C. Hamaker, The London-van der Waals attraction between spherical particles, Physica 4 (10) (1937) 1058-1072.

[36] E.M. Lifshitz, The theory of molecular attractive forces between solids, Soviet Physics 2 (1) (1956) 73-83. 